\title{
Probing leptoquark production at IceCube
}

\author{
Luis A. Anchordoqui, ${ }^{1}$ Carlos A. García Canal, ${ }^{2}$ Haim Goldberg, ${ }^{3}$ Daniel Gomez Dumm, ${ }^{2}$ and Francis Halzen ${ }^{4}$ \\ ${ }^{1}$ Department of Physics, University of Wisconsin-Milwaukee, P.O. Box 413, Milwaukee, Wisconsin 53201, USA \\ ${ }^{2}$ IFLP (CONICET) Dpto. de Física, Universidad Nacional de La Plata, C.C.67, La Plata (1900), Argentina \\ ${ }^{3}$ Department of Physics, Northeastern University, Boston, Massachusetts 02115, USA \\ ${ }^{4}$ Department of Physics, University of Wisconsin, Madison, Wisconsin 53706, USA
}

(Received 2 October 2006; published 29 December 2006)

\begin{abstract}
We emphasize the inelasticity distribution of events detected at the IceCube neutrino telescope as an important tool for revealing new physics. This is possible because the unique energy resolution at this facility allows to separately assign the energy fractions for emergent muons and taus in neutrino interactions. As a particular example, we explore the possibility of probing second and third generation leptoquark parameter space (coupling and mass). We show that production of leptoquarks with masses $\gtrsim 250 \mathrm{GeV}$ and diagonal generation couplings of $\mathcal{O}(1)$ can be directly tested if the cosmic neutrino flux is at the Waxman-Bahcall level.
\end{abstract}

DOI: 10.1103/PhysRevD.74.125021

PACS numbers: $13.15 .+\mathrm{g}, 95.85 . \mathrm{Ry}$

\section{INTRODUCTION}

Leptoquarks are $S U(3)$-colored particles which simultaneously carry nonzero baryon and lepton quantum numbers. They are predicted in several models (such as $S U(5)$ [1] or Pati-Salam $S U(4)$ [2]) addressing the unification of the lepton and quark sectors of the standard model (SM). In such models, the masses of the leptoquarks are generically superheavy, on the order of the GUT scale, which puts them out of reach of direct experimental access. Nevertheless, since leptoquarks with electroweak scale masses are not disallowed for any fundamental reason, it is of interest to conduct experimental searches to delimit their properties [3]. It is, of course, important to note that in order to avoid rapid baryon decay, the simultaneous trilinear coupling of the leptoquark to a purely hadronic channel needs to be excluded [4].

In general, the couplings of the leptoquark need not be generation-diagonal, and the problem of extracting limits on couplings and masses is complicated by the presence of a large-dimensional parameter space. Experiments at HERA have placed lower limits of $\mathcal{O}(300 \mathrm{GeV})$ on first generation leptoquark masses, for trilinear couplings of electroweak gauge strength [5]. Similar bounds, under the same assumptions, have been found at LEP from their search for anomalous 4-fermion vertices [6]. For first generation leptoquark trilinear couplings which are much smaller than gauge strength (as is the case for the Yukawas in the SM), the mass bounds are greatly weakened.

At the Tevatron, the leptoquarks could be produced in pairs, with identification made through decay topologies. In this way, the bounds are not dependent on the trilinear couplings, except for decay branching fractions. In the case of first [7] and second [8] generation leptoquarks, the final state topology consists of 2 hadronic jets + 2 charged leptons, and the resulting lower limits on the leptoquark mass are around $250 \mathrm{GeV}$. In the case of the third generation, a lower limit of $219 \mathrm{GeV}$ has been recently reported by the D0 Collaboration [9], by tagging on $2 b$ jets + missing energy. For this value of the leptoquark mass, the decay into $t \tau$ is largely suppressed compared to the $b \nu_{\tau}$ channel, so that the mass bound is nearly independent of even the branching fraction. As the explored mass region becomes larger, the $t \tau$ channel becomes more available and thus the mass limit obtained is pushed a bit lower (to $\geq 213 \mathrm{GeV}$ ).

In this work, we explore the possibility of probing second and third generation leptoquark parameter space (coupling and mass) with the IceCube neutrino detection facility [10]. This experiment, located below the surface of the Antarctic ice sheet at the geographic South pole, is required to be sensitive to the best estimates of potential cosmic ray neutrino fluxes. When completed, the telescope will consist of $80 \mathrm{~km}$-length strings, each instrumented with 6010 -inch photomultipliers spaced by $17 \mathrm{~m}$. The deepest module is $2.4 \mathrm{~km}$ below the surface. The strings are arranged at the apexes of equilateral triangles $125 \mathrm{~m}$ on a side. The instrumented (not effective) detector volume is a cubic kilometer. IceTop, a surface array of Cerenkov detectors deployed over $1 \mathrm{~km}^{2}$ above IceCube, augments the deep-ice component by providing a tool for calibration, background rejection, and air-shower physics. The expected energy resolution is \pm 0.1 on a $\log _{10}$ scale. Construction of the detector started in the Austral summer of 2004/2005 and will continue for 6 years, possibly less. At the present, data collection by the first 9 strings has begun.

The event signatures are grouped as tracks, showers, or a combination of the two. Tracks include muons resulting from both cosmic ray showers and from charged current (CC) interaction of muon neutrinos. Tracks can also be produced by $\tau$ leptons arising in ultrahigh energy $\nu_{\tau} \mathrm{CC}$ interactions. Showers are generated by neutrino collisions ( $\nu_{e}$ or low energy $\nu_{\tau}$ CC interactions, and all neutral 
current interactions) inside or near the detector, and by muon bremsstrahlung radiation near the detector.

The experimental situation is greatly simplified for neutrino energy $E_{\nu} \gtrsim 10^{6} \mathrm{GeV}$. A cut at this energy is sufficient to reduce the great majority of background from muon bremsstrahlung and tracks arising from muons produced in cosmic ray showers. Moreover, the flux of atmospheric neutrinos is low above this energy [11], so this cut generates a very pure sample of extraterrestrial neutrinos. Of particular interest here, for $E_{\nu}>10^{6} \mathrm{GeV}$, there is sufficient energy resolution ( \pm 0.2 on a $\log _{10}$ scale) to separately assign the energy fractions in the muon track and the hadronic shower, allowing the determination of the inelasticity distribution. Similarly, in the energy decade $10^{6.5}<E_{\nu} / \mathrm{GeV}<10^{7.5}$ one can expect good resolution (less than 5\%) in "double bang" events generated by incoming $\nu_{\tau}$ 's. Again, this will allow a reasonable measurement of the inelasticity distribution.

In this study, we emphasize the inelasticity $(y)$ distribution of events as an important tool for detection of new physics. In particular, we will find that the $y$ distribution of events generated through resonant leptoquark production differs substantially from the SM prediction. If the event rate for the new physics turns out to be comparable to SM expectations, then the $y$ profile of the measured data can be used to probe the coupling-mass leptoquark parameter space. The outline of the paper is as follows: In Sec. II we derive the relevant $y$-distribution of events generated through production and decay of a scalar leptoquark under the assumption of diagonal generation coupling. Armed with this distribution, in Sec. III we present a statistical method for assessing the significance of discovery criteria. Our conclusions are collected in Sec. IV.

\section{LEPTOQUARK PHENOMENOLOGY}

A general Lagrangian for $S U(3)_{C} \otimes S U(2)_{L} \otimes$ $U(1)_{Y}$-invariant flavor-diagonal leptoquark couplings has been presented in [12]. To illustrate our proposal, we consider the simple case of $S U(2)$-singlet scalar leptoquarks $S_{i}$ which interact with quarks and leptons through the Lagrangian

$$
\mathfrak{\Omega}_{L Q}=\sum_{i}\left(g_{L} \bar{Q}_{i L}^{c} i \tau_{2} L_{i L}+g_{R} \bar{u}_{i R}^{c} l_{i R}\right) S_{i}
$$

Here $Q_{i}=\left(u_{i} d_{i}\right)^{T}$ and $L_{i}=\left(\nu_{i} l_{i}\right)^{T}$ stand for quark and lepton $S U(2)$ left-handed doublets, $u_{i R}$ and $l_{i R}$ are righthanded singlets, and $g_{L}$ and $g_{R}$ are the corresponding coupling constants. Subindices $i$, running from 1 to 3, label the quark or fermion family. For simplicity we will assume that the interaction conserves leptoquark family quantum numbers separately (i.e., there is no mixing between different families). Thus in the following subindices $i$ will be dropped, and uplike and downlike quarks will be denoted generically with $U$ and $D$, respectively.
We will be considering the inclusive $\nu P$ scattering schematically shown in Fig. 1. The crosshatched circle includes both resonant leptoquark production and decay, as well as $u$-channel exchange of a leptoquark leading to the same final state. We will not be considering couplings $g_{L(R)} \gtrless 2$, since such a coupling could lead to Landau singularities at low energies. Hence, we will assume that the resonant cross section largely dominates the process, and the narrow width $\delta$-function approximation will be valid.

Let us assume that an incoming neutrino collides with a proton target with center-of-mass energy $s$. If the neutrino hits a downlike parton $D$, the inclusive cross section for the process shown in Fig. 1 in the parton model is given by

$$
\frac{d \sigma_{L Q}\left(\nu P \rightarrow l^{-} X\right)}{d^{3} k^{\prime} / E^{\prime}}=\int d x D(x) \frac{d \hat{\sigma}_{L Q}\left(\nu D \rightarrow l^{-} U\right)}{d^{3} k^{\prime} / E^{\prime}}
$$

where $D(x)$ is the corresponding parton distribution function (pdf). The neutrino-parton cross section reads

$$
\begin{aligned}
\frac{d \hat{\sigma}_{L Q}\left(\nu D \rightarrow l^{-} U\right)}{d^{3} k^{\prime} / E^{\prime}}= & \frac{1}{(2 \pi)^{2}} \frac{1}{2 \mathcal{F}} \int d^{4} p^{\prime} \\
& \times \delta^{4}\left(k+p-k^{\prime}-p^{\prime}\right) \delta^{+}\left(p^{\prime 2}-m_{u}^{2}\right) \\
& \times \frac{1}{2} \sum_{\text {spin }}\left(\left|\mathfrak{M}_{L}\right|^{2}+\left|\mathfrak{M}_{R}\right|^{2}\right)
\end{aligned}
$$

where $\mathcal{F}=2 x s=2 \hat{s}$ is the invariant flux, $m_{U}$ is the mass of the outgoing uplike quark, and $E^{\prime}$ is the lab energy of the outgoing charged lepton. In the resonant approximation, the amplitude for the production of a left-handed charged lepton is given by

$$
\mathfrak{M}_{L}=g_{L}^{2} \frac{\bar{l}_{L}\left(k^{\prime}\right) U_{L}^{c}\left(p^{\prime}\right) \bar{D}_{L}^{c}(p) \nu_{L}(k)}{\hat{s}-M^{2}-i \Gamma M},
$$

where $M$ and $\Gamma$ are the mass and width of the leptoquark. A similar expression, replacing $g_{L}^{2} \rightarrow g_{L} g_{R}$, holds for the decay through the right-handed channel. It is easy to see that there is no interference between $\mathfrak{M}_{L}$ and $\mathfrak{M}_{R}$.

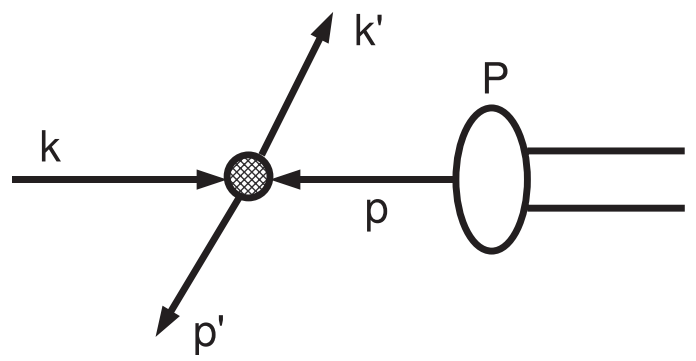

FIG. 1. Schematic diagram of a neutrino-parton collision, in which a neutrino with momentum $k$ hits a quark with momentum $p=x P$ giving rise to a secondary charged lepton and quark with momenta $k^{\prime}$ and $p^{\prime}$, respectively. Here $x$ is the fractional energy of the struck parton in the nucleon having momentum $P$. 
Now in the narrow resonance approximation one has

$$
\frac{1}{\left(\hat{s}-M^{2}\right)^{2}+(\Gamma M)^{2}} \rightarrow \frac{\pi}{\Gamma M} \delta\left(\hat{s}-M^{2}\right) .
$$

Then, after summing over spins of outgoing fermions one arrives to

$$
\begin{aligned}
\frac{1}{2} \sum_{\text {spin }}\left(\left|\mathfrak{M}_{L}\right|^{2}+\left|\mathfrak{M}_{R}\right|^{2}\right)= & \frac{\pi}{2} g_{L}^{2}\left(g_{L}^{2}+g_{R}^{2}\right) \frac{\hat{s}\left(\hat{s}-m_{U}^{2}\right)}{M \Gamma} \\
& \times \delta\left(\hat{s}-M^{2}\right),
\end{aligned}
$$

where we have neglected both the mass of the downlike quark and the mass of the outgoing charged lepton. We have kept instead the $m_{U}$ dependence, since it is relevant in the case of the third family, where the outgoing quark would be a top. As a further assumption, we will consider that the leptoquark width is dominated by the $U l$ and $D \nu$ quark-lepton channels. This leads to

$$
\Gamma=\frac{M}{16 \pi}\left\{g_{L}^{2}\left[\left(1-\lambda_{U}\right)^{2}+1\right]+g_{R}^{2}\left(1-\lambda_{U}\right)^{2}\right\},
$$

where $\lambda_{U}=m_{U}^{2} / M^{2}$. Substituting Eqs. (3), (6), and (7) into Eq. (2) yields

$$
\begin{aligned}
\frac{d \sigma_{L Q}^{(\alpha)}}{d^{3} k^{\prime} / E^{\prime}}= & \frac{g_{L}^{2}\left(g_{L}^{2}+g_{R}^{2}\right)\left(1-\lambda_{U}\right)}{\left(g_{L}^{2}+g_{R}^{2}\right)\left(1-\lambda_{U}\right)^{2}+g_{L}^{2}} \int d x \frac{D(x)}{2 x s} M^{2} \\
& \times \delta\left(Q^{2}-2 m_{p} y E_{\nu} x+m_{U}^{2}\right) \delta\left(x s-M^{2}\right) \\
= & \frac{1}{2 s} \frac{g_{L}^{2}\left(g_{L}^{2}+g_{R}^{2}\right)\left(1-\lambda_{U}\right)}{\left(g_{L}^{2}+g_{R}^{2}\right)\left(1-\lambda_{U}\right)^{2}+g_{L}^{2}} D\left(M^{2} / s\right) \\
& \times \delta\left(Q^{2}-y M^{2}+m_{U}^{2}\right),
\end{aligned}
$$

where $Q^{2}=-\left(k-k^{\prime}\right)^{2}$, and the inelasticity $y$ is defined as $y=\left(E_{\nu}-E^{\prime}\right) / E_{\nu}, E_{\nu}$ being the lab energy of the incoming neutrino. Indices $\alpha=1,2,3$ correspond to the uplike quarks $U=u, c, t$, respectively. After adequate changes of variables and integrations [13] we find

$$
\frac{d \sigma_{L Q}^{(\alpha)}}{d y}=\frac{\pi}{2} \frac{g_{L}^{2}\left(g_{L}^{2}+g_{R}^{2}\right)\left(1-\lambda_{U}\right)}{\left(g_{L}^{2}+g_{R}^{2}\right)\left(1-\lambda_{U}\right)^{2}+g_{L}^{2}} \frac{D\left(M^{2} / s\right)}{s} .
$$

The inelasticity $y$ lies in the range $\lambda_{U} \leq y \lessgtr 1$. Note that the $y$ distribution of the resonant process is approximately flat (at the energies of interest, the $Q^{2}$ dependence of the pdf can be neglected), in contrast to the characterizing SM charged current $(\mathrm{CC})$ processes in which

$$
\begin{aligned}
\frac{d \sigma_{\mathrm{SM}}^{\mathrm{CC}}}{d y}= & \frac{2 G_{\mathrm{F}}^{2} m_{p} E_{\nu}}{\pi}\left(\frac{M_{W}^{2}}{Q^{2}+M_{W}^{2}}\right)^{2} \int d x\left[x q\left(x, Q^{2}\right)\right. \\
& \left.+x \bar{q}\left(x, Q^{2}\right)(1-y)^{2}\right],
\end{aligned}
$$

where $G_{\mathrm{F}}=1.16632 \times 10^{-5} \mathrm{GeV}^{-2}$ is the Fermi constant, $M_{W}$ is the mass of the $W$ gauge boson, and $q(x)$ and $\bar{q}(x)$ stand for combinations of quark and antiquark proton pdf's, respectively [14]. The $y$ dependence of the SM cross section is shown in Fig. 6 of Ref. [15]. In the next section we exploit the differing $y$ dependences of the leptoquark and SM interactions to constrain the parameter space of the new physics.

\section{SENSITIVITY REACH AT ICECUBE}

To evaluate the prospects for probing leptoquark production at IceCube, one has to estimate the "beam luminosity," i.e. the magnitude of the (yet to be detected) neutrino flux. We know that cosmic accelerators produce particles with energies in excess of $10^{11} \mathrm{GeV}$ (we do not know where or how [16]), and a neutrino beam is expected to come in association with these cosmic rays [17]. However, given our ignorance of the opacity of the sources, it is difficult to calculate the magnitude of the neutrino flux. The usual benchmark here is the so-called WaxmanBahcall (WB) flux

$$
E_{\nu}^{2} \phi_{\mathrm{WB}}^{\nu}\left(E_{\nu}\right) \simeq 6 \times 10^{-8} \mathrm{GeV} \mathrm{cm}^{-2} \mathrm{~s}^{-1} \mathrm{sr}^{-1}
$$

(all flavors), which is derived assuming that neutrinos come from transparent cosmic ray sources [18], and that there is an adequate transfer of energy to pions following $p p$ collisions. Here we will rely on this expression to estimate the event rates needed to quantify the IceCube sensitivity to leptoquark production. However, one should keep in mind that if there are in fact "hidden" sources which are opaque to ultrahigh energy cosmic rays, then the expected neutrino flux will be higher [19]. Moreover, if the extragalactic cosmic rays begin to dominate over the galactic component at energies as low as $\sim 10^{9} \mathrm{GeV}$, as suggested recently [20], then the required power of the extragalactic sources will increase by a factor of $\sim 2$, implying a concomitantly larger neutrino flux [21].

IceCube is sensitive to both downward and upward coming cosmic neutrinos. However, to remain conservative with our statistical sample, here we select only downward going events. To a good approximation, the expected number of such events at IceCube is given by

$$
\mathcal{N}=2 \pi n_{\mathrm{T}} T \int d E_{\nu} \sigma_{\text {tot }}\left(E_{\nu}\right) \phi_{\mathrm{WB}}^{\nu}\left(E_{\nu}\right),
$$

where $n_{\mathrm{T}}$ is the number of target nucleons in the effective volume, $T$ is the running time, and $\sigma_{\text {tot }}\left(E_{\nu}\right)$ is the total neutrino-nucleon cross section. In our analysis we are interested only in CC contained events, for which an accurate measurement of the inelasticity can be obtained. The IceCube's effective volume for (background rejected) contained events is roughly $1 \mathrm{~km}^{3}$ [22], which corresponds to $n_{\mathrm{T}} \simeq 6 \times 10^{38}$.

Hereafter we focus on neutrino energies in the range $10^{7}<E_{\nu} / \mathrm{GeV}<10^{7.5}$, where the background from atmospheric neutrinos is negligible, but the extraterrestrial flux is expected to be significant. Thus, we will consider a medium energy $\left\langle E_{\nu}\right\rangle=10^{7.25} \mathrm{GeV}$. At production, the WB flux has flavor ratios $\nu_{\mu}: \nu_{e}: \nu_{\tau}=2: 1: 0$, but this quickly transforms to $1: 1: 1$ through neutrino oscillations 
[23]. One has then

$$
\phi_{\mathrm{WB}}^{\nu_{\alpha}}\left(\left\langle E_{\nu}\right\rangle\right) \simeq 6 \times 10^{-23} \mathrm{GeV}^{-1} \mathrm{~cm}^{-2} \mathrm{~s}^{-1} \mathrm{sr}^{-1}
$$

for each flavor $\alpha$. Now it is possible to increase the ratio signal/background events by performing a cut in the inelasticity $y$. Given the dependence on $y$ of the $\sigma_{\mathrm{SM}}^{\mathrm{CC}}$ cross section, the flat behavior of the $\sigma_{L Q}$ cross section, and the available phase space for quark production, it is convenient to consider events with relatively large values of $y$. We choose here events in the range $y \geq 0.5$. With this cut, the integration of Eq. (10) leads to

$$
\left.\sigma_{\mathrm{SM}}^{\mathrm{CC}}\left(\left\langle E_{\nu}\right\rangle\right)\right|_{y \geq 0.5} \simeq 8 \times 10^{-34} \mathrm{~cm}^{2} .
$$

One has to take into account that this result carries a systematic error of about $20 \%[14,24]$ due to uncertainties in the extrapolation of the pdf's [25]. For illustrative comparison the second generation leptoquark cross section in the high $y$ region is calculated from Eq. (9) to be

$$
\left.\sigma_{L Q}\left(\left\langle E_{\nu}\right\rangle\right)\right|_{y \geq 0.5} \simeq 2 \times 10^{-33} \mathrm{~cm}^{2},
$$

for fiducial values $g_{L}=g_{R}=1$ and leptoquark mass $M=$ $300 \mathrm{GeV}$. From Eqs. (12) and (13) we can now easily estimate the number of expected SM background events during the lifetime of the experiment. Taking $T=15 \mathrm{yr}$, for each neutrino flavor $\alpha$ one has

$$
\left.\mathcal{N}_{\mathrm{B}}^{(\alpha)} \simeq 2 \pi n_{\mathrm{T}} T \sigma_{\mathrm{SM}}^{\mathrm{CC}}\left(\left\langle E_{\nu}\right\rangle\right)\right|_{y \geq 0.5} \phi_{\mathrm{WB}}^{\nu_{\alpha}}\left(\left\langle E_{\nu}\right\rangle\right) \Delta E_{\nu}=2,
$$

where $\quad \Delta E_{\nu}=10^{7.5} \mathrm{GeV}-10^{7} \mathrm{GeV} \simeq 2.2 \times 10^{7} \mathrm{GeV}$. (Note that the background is cosmic neutrinos. Today's signal, tomorrow's background.) In the same way, the number of signal events $\mathcal{N}_{\mathrm{S}}$ will be approximately given by

$$
\left.\mathcal{N}_{\mathrm{S}}^{(\alpha)} \simeq 2 \pi n_{\mathrm{T}} T \sigma_{L Q}\left(\left\langle E_{\nu}\right\rangle\right)\right|_{y \geq 0.5} \phi_{\mathrm{WB}}^{\nu_{\alpha}}\left(\left\langle E_{\nu}\right\rangle\right) \Delta E_{\nu},
$$

which for the above proposed leptoquark interaction is just a function of the couplings $g_{R, L}$ and the leptoquark mass $M$.

It should be noted that the neutrino induced events do not constitute the sole background. As mentioned in the introduction there are muons (produced in the atmosphere) which traverse the detector and may deposit energy through bremsstrahlung radiation. In our energy bin, one may expect 10 muon traversals in 15 years. However, our inelasticity cut will completely eliminate this source of background, because of the negligible probability for muons to radiate $50 \%$ of their energy.

To determine the bounds for leptoquark production, let us assume that $2 \nu_{\alpha}$-events are in fact observed with $y \geq$ 0.5. Then, at $90 \%$ C.L., there will be an upper bound on signal events given by $\mathcal{N}_{\mathrm{S}}^{(\alpha)} \leq 3.91$ [26]. For simplicity, we consider the left-right symmetric case in which $g_{L}=$ $g_{R}$. Then, after numerical evaluation of the leptoquark cross sections, the upper bounds on $\mathcal{N}_{\mathrm{S}}^{(\alpha)}$ can be translated

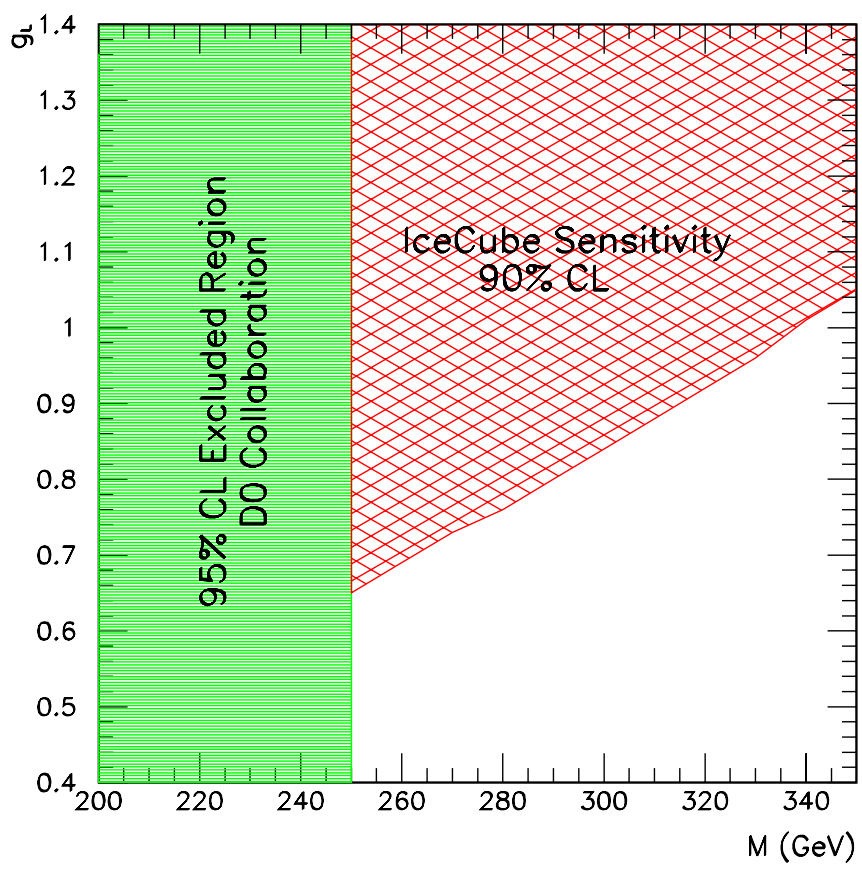

FIG. 2 (color online). Sensitivity reach of IceCube (90\% C.L.) to probe second generation $S U(2)$-singlet scalar leptoquark parameter space (coupling and mass). For comparison, the existing limit (95\% C.L.) reported by D0 Collaboration [8] is also shown.

into contours of constant likelihood in the $M-g_{L}$ plane. Our results are displayed in Figs. 2 and 3, where we show the

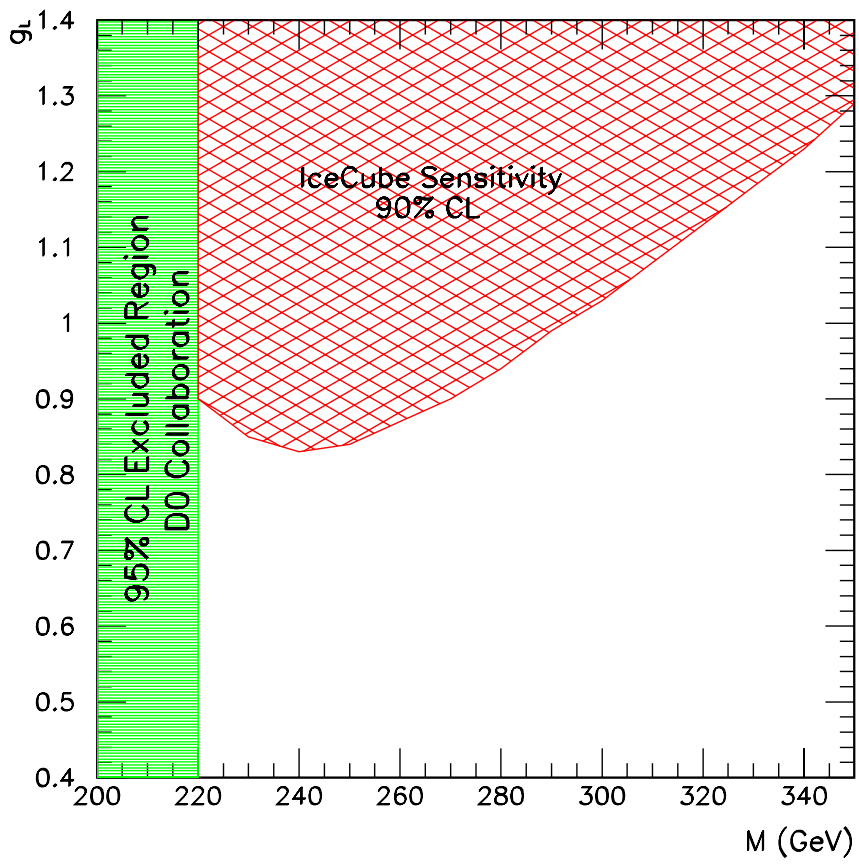

FIG. 3 (color online). Sensitivity reach of IceCube (90\% C.L.) to probe third generation $S U(2)$-singlet scalar leptoquark parameter space (coupling and mass). For comparison, the existing limit (95\% C.L.) reported by D0 Collaboration [9] is also shown. 
sensitivity reach of IceCube together with the existing limits from D0 Collaboration $[8,9]$.

In the case of the third family, it can be seen that the sensitivity is maximal for leptoquarks of $M \simeq 245 \mathrm{GeV}$. For lower leptoquark masses (in the narrow resonance limit) the allowed inelasticity range - and thus the leptoquark cross section-becomes reduced due to phase space suppression, owing to the large mass of the top quark.

In order to estimate the significance of the assumption $g_{L}=g_{R}$, we have also considered the case of purely lefthanded leptoquark currents, i.e. $g_{R}=0$. By looking at Eq. (9), it can be seen that this implies an average reduction in the leptoquark cross section by a factor of about 0.75 and 0.65 for the second and third families, respectively.

\section{CONCLUSIONS}

In this paper we have introduced the measurement of inelasticity as a powerful tool for probing new physics in cosmic neutrino interactions. As an illustrative example, we have discussed the possibility of detecting leptoquark production at the IceCube neutrino telescope [27]. We estimated the expected event rate at IceCube to be comparable to the one predicted for cosmic ray facilities that make use of the atmosphere as the detector calorimeter [28]. However, the ability of IceCube to accurately measure the inelasticity distribution of events provides a unique method for SM background rejection, allowing powerful discrimination of resonant processes: we have shown that production of leptoquarks with masses $\gtrsim$ $250 \mathrm{GeV}$ and diagonal generation couplings of $\mathcal{O}(1)$ can be directly tested at the Antarctic ice cap.

In closing, some comments are in order. First, we have not taken account of any systematic considerations concerning the detector-these are beyond the scope of the present work. Second, for reasons of simplicity, we have not included upcoming events close to the horizon. Although these Earth-skimming neutrinos have the potential of nearly doubling our signal event background (and thus nearly halving the required observation time scale), their proper consideration will require a full Monte Carlo simulation.

\section{ACKNOWLEDGMENTS}

C.A.G.C. is supported by CONICET and ANPCyT, Argentina. H. G. is supported by the U.S. National Science Foundation (NSF) Grant No. PHY-0244507. D. G.D. is supported by CONICET and ANPCyT, Argentina, under Grant No. PIP 6009, No. PICT04 0325374, and No. PICT02-03-10718. F. H. is supported in part by the U.S. NSF under Grant No. OPP-0236449, in part by the U.S. Department of Energy (DoE) Grant No. DE-FG02-95ER40896, and in part by the University of Wisconsin Research Committee with funds granted by the Wisconsin Alumni Research Foundation.
[1] H. Georgi and S. L. Glashow, Phys. Rev. Lett. 32, 438 (1974).

[2] J. C. Pati and A. Salam, Phys. Rev. D 10, 275 (1974).

[3] A theoretical framework with light leptoquarks has been discussed in, I. Dorsner and P. F. Perez, Nucl. Phys. B723, 53 (2005); I. Dorsner, P. F. Perez, and R. Gonzalez Felipe, Nucl. Phys. B747, 312 (2006).

[4] I. Hinchliffe and T. Kaeding, Phys. Rev. D 47, 279 (1993); A. Y. Smirnov and F. Vissani, Phys. Lett. B 380, 317 (1996); G. Bhattacharyya and P. B. Pal, Phys. Lett. B 439, 81 (1998).

[5] C. Adloff et al. (H1 Collaboration), Phys. Lett. B 568, 35 (2003); A. Aktas et al. (H1 Collaboration), Phys. Lett. B 629, 9 (2005).

[6] G. Abbiendi et al. (OPAL Collaboration), Eur. Phys. J. C 6, 1 (1999); P. Abreu et al. (DELPHI Collaboration), Phys. Lett. B 446, 62 (1999); M. Acciarri et al. (L3 Collaboration), Phys. Lett. B 489, 81 (2000).

[7] B. Abbott et al. (D0 Collaboration), Phys. Rev. Lett. 79, 4321 (1997); F. Abe et al. (CDF Collaboration), Phys. Rev. Lett. 79, 4327 (1997); V. M. Abazov et al. (D0 Collaboration), Phys. Rev. D 71, 071104 (2005).

[8] V. M. Abazov et al. (D0 Collaboration), Phys. Lett. B 636, 183 (2006)
[9] D0 Collaboration, Report No. D0 Note 5176-CONF; V. M. Abazov (D0 Collaboration), Phys. Lett. B 640, 230 (2006).

[10] J. Ahrens et al. (IceCube Collaboration), Nucl. Phys. B, Proc. Suppl. 118, 371 (2003).

[11] P. Lipari, Astropart. Phys. 1, 195 (1993).

[12] W. Buchmuller, R. Ruckl, and D. Wyler, Phys. Lett. B 191, 442 (1987); 448, 320(E) (1999).

[13] F. Halzen and A. D. Martin, Quarks And Leptons: An Introductory Course In Modern Particle Physics (John Wiley \& Sons, New York, 1984) p. 194.

[14] R. Gandhi, C. Quigg, M. H. Reno, and I. Sarcevic, Phys. Rev. D 58, 093009 (1998).

[15] R. Gandhi, C. Quigg, M.H. Reno, and I. Sarcevic, Astropart. Phys. 5, 81 (1996).

[16] L. Anchordoqui, T. Paul, S. Reucroft, and J. Swain, Int. J. Mod. Phys. A 18, 2229 (2003).

[17] T. K. Gaisser, F. Halzen, and T. Stanev, Phys. Rep. 258, 173 (1995); 271, 355(E) (1996); J. G. Learned and K. Mannheim, Annu. Rev. Nucl. Part. Sci. 50, 679 (2000); F. Halzen and D. Hooper, Rep. Prog. Phys. 65, 1025 (2002).

[18] E. Waxman and J. N. Bahcall, Phys. Rev. D 59, 023002 (1998). 
[19] F. W. Stecker, C. Done, M. H. Salamon, and P. Sommers, Phys. Rev. Lett. 66, 2697 (1991); 69, 2738(E) (1992); A. Neronov, D. Semikoz, F. Aharonian, and O. Kalashev, Phys. Rev. Lett. 89, 051101 (2002); F. W. Stecker, Phys. Rev. D 72, 107301 (2005).

[20] V. Berezinsky, A.Z. Gazizov, and S. I. Grigorieva, Phys. Rev. D 74, 043005 (2006); R. U. Abbasi et al. (High Resolution Fly's Eye Collaboration), Phys. Rev. Lett. 92, 151101 (2004); V. Berezinsky, A. Z. Gazizov, and S. I. Grigorieva, Phys. Lett. B 612, 147 (2005).

[21] M. Ahlers, L. A. Anchordoqui, H. Goldberg, F. Halzen, A. Ringwald, and T.J. Weiler, Phys. Rev. D 72, 023001 (2005).

[22] L. Anchordoqui and F. Halzen, Ann. Phys. (N.Y.) 321, 2660 (2006).

[23] J. G. Learned and S. Pakvasa, Astropart. Phys. 3, 267 (1995).

[24] G. M. Frichter, D. W. McKay, and J. P. Ralston, Phys. Rev.
Lett. 74, 1508 (1995); 77, 4107(E) (1996); L. A. Anchordoqui, A. M. Cooper-Sarkar, D. Hooper, and S. Sarkar, Phys. Rev. D 74, 043008 (2006).

[25] J. Pumplin, D. R. Stump, J. Huston, H. L. Lai, P. Nadolsky, and W. K. Tung, J. High Energy Phys. 07 (2002) 012; R. S. Thorne, A. D. Martin, W. J. Stirling, and R. G. Roberts, hep-ph/0407311; S. Chekanov et al. (ZEUS Collaboration), Phys. Rev. D 67, 012007 (2003).

[26] G. J. Feldman and R. D. Cousins, Phys. Rev. D 57, 3873 (1998).

[27] Previous approaches in this direction have been carried out in V.S. Berezinsky, Yad. Fiz. 41, 393 (1985); R. W. Robinett, Phys. Rev. D 37, 84 (1988); M. A. Doncheski and R. W. Robinett, Phys. Rev. D 56, 7412 (1997); M. Carena, D. Choudhury, S. Lola, and C. Quigg, Phys. Rev. D 58, 095003 (1998).

[28] M. C. Espirito Santo, A. Onofre, M. Paulos, M. Pimenta, J. C. Romao, and B. Tome, J. Phys. G 32, 609 (2006). 